Natural and Applied Sciences International Journal (NASIJ)

https://doi.org/10.47264/idea.nasij/ 1.1.1

Vol. 1, No. 1, (January-December 2020), 01-10

https://www.ideapublishers.org/index.php/nasij

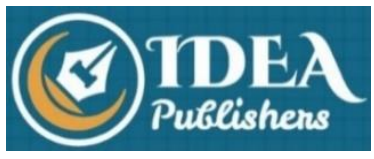

Research Article

\title{
Comparison of GPS TEC with IRI Models of 2007, 2012 and 2016 over Sukkur, Pakistan
}

Adil Hussain $^{1} \&$ Munawar Shah*1-2

1. Department of GNSS, Institute of Space Technology, Islamabad, Pakistan.

2. Space Education and GNSS Lab, National Center of GIS and Space Application, Institute of Space Technology, Islamabad, Pakistan.

* Corresponding Author Email: munawar.shah@mail.ist.edu.pk; shahmunawar1@gmail.com

\begin{abstract}
The international reference ionosphere (IRI) models have been widely used for correcting the ionospheric scintillations at different altitude levels. An evaluation on the performance of VTEC correction from IRI models (version 2007, 2012 and 2016) over Sukkur, Pakistan $\left(27.71^{\circ} \mathrm{N}\right.$, $68.85^{\circ} \mathrm{E}$ ) is presented in this work. Total Electron Content (TEC) from IRI models and GPS in 2019 over Sukkur region are compared. The main aim of this comparative analysis is to improve the VTEC in low latitude Sukkur, Pakistan. Moreover, this study will also help us to identify the credible IRI model for the correction of Global Positioning System (GPS) signal in low latitude region in future. The development of more accurate TEC finds useful applications in enhancing the extent to which ionospheric influences on radio signals are corrected. VTEC from GPS and IRI models are collected between May 1, 2019 and May 3, 2019. Additionally, Dst and Kp data are also compared in this work to estimate the geomagnetic storm variations. This study shows a good correlation of 0.83 between VTEC of GPS and IRI 2016. Furthermore, a correlation of 0.82 and 0.78 is also recorded for IRI 2012 and IRI 2007 respectively, with VTEC of GPS. The IRI TEC predictions and GPS-TEC measurements for the studied days reveal the potential of IRI model as a good candidate over Pakistan.
\end{abstract}

Keywords: GPS TEC, IRI Models, Ionosphere, Geomagnetic Storm, Delays, Sukkur.

\section{How to Cite:}

Hussain, A. \& Munawar, S. (2020). Comparison of GPS TEC with IRI Models of 2007, 2012 and 2016 over Sukkur, Pakistan. Natural \& Applied Sciences International Journal (NASIJ), 1(1), 01-00. https://doi.org/10.47264/idea.nasij/1.1.1

\section{Publisher's Note:}

IDEA PUBLISHERS (IDEA Journals Group) stands neutral regarding jurisdictional claims in the published maps and institutional affiliations.

\section{Copyright:}

(C) 2020 The Author(s), published by IDEA PUBLISHERS (IDEA Journals Group)

This is an Open Access article published under the Creative Commons Attribution-NonCommercial 4.0 International License (http://creativecommons.org/licenses/by-nc/4.0/) 


\section{Introduction}

A geomagnetic storm is a temporary disturbance of the Earth's magnetosphere caused by a solar wind shock wave and/or cloud of magnetic field that interacts with the Earth's magnetic field. The disturbance that drives the magnetic storm may be a solar Coronal Mass Ejection (CME) or a co-rotating interaction region (CIR), a high-speed stream of solar wind originating from a coronal hole. Frequency of geomagnetic storm can increase and decrease with sunspot cycle. During solar maximum, geomagnetic storms occur more often, with the majority driven by CMEs. During solar minimum, storms are mainly driven by CIRs. The increase in the solar wind pressure initially compresses the magnetosphere. The solar wind's magnetic field interacts with the Earth's magnetic field and transfers an increased energy into the magnetosphere. Both interactions cause an increase in plasma movement and an increase in the electric current in the magnetosphere and ionosphere. During the main phase of a geomagnetic storm, electric current in the magnetosphere creates a magnetic force that pushes out the boundary between the magnetosphere and the solar wind.

The ionosphere is a part of the Earth's atmosphere, which extends from approximately 50 $1000 \mathrm{~km}$ in altitude. It contains a significant number of free electrons and ions that can influence the radio wave propagation. The Sun's ultraviolet and X-ray emissions are primarily responsible for the ionization in ionosphere. When a signal from the GPS satellite is travelling through the ionized layer in the ionosphere, refraction, or bending of the wave occurs. The amount of refraction that occurs depends on three main factors: (1) the density of ionization of the layer, (2) the frequency of the radio wave, e.g., for GPS L1 it is $1575.42 \mathrm{MHz}$, and (3) the angle at which the wave enters the layer. An important descriptive quantity in describing the effect of the ionosphere on the GPS signal is the total electron content (TEC). TEC is the total number of electron present along a path between the satellite and the receiver on Earth, with units of electrons per square meter, where $10^{16}$ electrons $/ \mathrm{m}^{2}=1$ TEC unit (TECU). Geomagnetic storms are major factors disturbing TEC. It is necessary to measure TEC to minimize the errors and delays in GNSS signals. TEC can be measured through a GPS receiver using different formulas and TEC can also be modelled using IRI models.

This paper presents a comparison between GPS TEC and TEC from IRI models of 2007, 2012 and 2016 over Sukkur Pakistan between May 1, 2019 and May 3, 2019. IRI models are globally accepted for specifying ionospheric parameters. Lot of work is done on different IRI models to specify ionospheric parameters. All IRI models give us almost accurate data of ionospheric parameters so these models are widely used all over the world. Some of the latest studies on IRI models is discussed below. Okoh et al. (2012) used IRI TEC data to compare it with GPS TEC in 2012 over Nsukka, Nigeria. Tariq et al. (2019) used IRI TEC to compare it with GPS TEC for different region of Pakistan in the year 2019. Tariq et al. (2020) also used IRI TEC to compare it with GPS TEC for different regions of Turkey. Lot of work is done by other researchers on IRI models. Most of them have suggested IRI models to specify different ionospheric parameters because all of them found IRI models as a good tool for specifying ionospheric parameters. Previous researches have compared GPS TEC with only one IRI model. In this research we have compared GPS TEC with three IRI models over low latitude region to find out the best IRI model for this specific area.

\section{Data and Methodology}

In this paper, we compared the measured VTEC with modelled VTEC from IRI model of 2007, 
2012 and 2016. The International Reference Ionosphere (IRI) model has been accepted as a defector standard for specifying ionospheric parameters across the globe. In this paper, we present an evaluation of the model's performance over Sukkur, Pakistan. Sukkur is a low latitude region and Pakistan's very first multi frequency GPS receiver is installed in IBA University, Sukkur.

The IRI is a working group, jointly sponsored by the Committee on Space Research (COSPAR) and the International Union of Radio Science (URSI) that develops an empirical ionospheric model to correct the measured VTEC. The IRI model gives spatial and temporal representations of ionospheric parameters (including TEC) and has been widely regarded as the international standard for specifying ionospheric parameters (Bilitza, 2001; Bilitza et al., 1999). In this work, we use IRI-2007, IRI-2012 and IRI 2016 model's data that is taken in 1st, 2nd and 3rd of May 2019. In general, the standard IRI options are used, and the upper integration limit for the TEC values was set at $2000 \mathrm{~km}$, a good limit for the IRI models corresponding data from the GPS equipment also excludes ionospheric TEC contribution.

For this research, GPS TEC from Sukkur GPS station ( $1^{\text {st }}$ ever installed multi frequency GNSS receiver in Pakistan for research purpose) and different storm indices are calculated for first three days of May 2019 ( $1^{\text {st }}$ May 2019 to $3^{\text {rd }}$ May 2019). Data of geomagnetic indices of atmosphere are taken from NASA OMNI web through the link (https://data.nasa.gov/SpaceScience/OMNIWeb-Plus/).

TEC is an indicator of ionospheric variability that is derived by the improved GPS signal through free electrons. The range error in GPS signal is proportional to the integral of electron density (TEC) along the ray path. The TEC taken from the ground-based Sukkur station of GPS can be explained as TEC is measured in units' of $10^{16}$ electrons meter per square area, where 1 TEC unit $\left(\right.$ TECU) $=10^{16} \mathrm{el} / \mathrm{m}^{2}$ (Shah et al., 2020a; Shah et al., 2020b; Shah et al., 2020c).

The slant TEC is obtained from the Sukkur GPS stations in the on first three days of May 2019. It can be measured by the number of electrons in $(1 \times 1) \mathrm{m}^{2}$ tube along the ray path of an observed signal, which is described in the unit of TEC as explained above. The STEC from the dual-frequency receiver is measured by the following equations in (Jin et al., 2017):

$S T E C=\frac{f_{1}^{2} f_{2}^{2}}{40.28\left(f_{1}^{2}-f_{2}^{2}\right)}\left(L_{1}-L_{2}+\lambda_{1}\left(N_{1}-N_{2}\right)-\lambda_{2}\left(N_{1}-N_{2}\right)+€\right)$

$S T E C=\frac{f_{1}^{2} f_{2}^{2}}{40.28\left(f_{1}^{2}-f_{2}^{2}\right)}\left(P_{1}-P_{2}-\left(d_{1-} d_{1}\right)+€\right)$

Here $f_{1}$ and $f_{2}$ are carrier phase frequencies of GNSS, $P$ and $L$ are the pseudo-range and carrier phase observation of delay path of GNSS signal, $\lambda$ is the wavelength of GNSS signal, $N$ is the ambiguity of GNSS signal, $b$ is the instrumental biases, $d$ is the differential code bias, €is the random residual along the ray path.

Moreover, STEC is transformed into the vertical TEC (VTEC) by following function shown by equation (3):

$V T E C=S T E C \cdot \cos \left(\arcsin \left(\frac{R \cdot \sin Z}{R+H}\right)\right)$ 
$\mathrm{R}$ is the Earth's radius, $\mathrm{H}$ is the height of the top layer of the ionosphere in atmospheric altitude, and $\mathrm{Z}$ is the satellite elevation angle for pierce point (Shah et al. 2020a).

Correlation coefficient is measured using equation 4 for GPS TEC all IRI models and the data is compared.

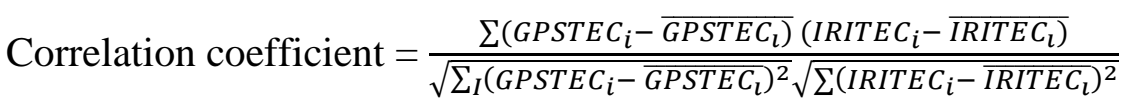

To evaluate the model performance in terms of daily fluctuations, the diurnal hourly VTEC are selected for three days in the month of May at the Sukkur stations from in 2019. In addition, to analyze the daily VTEC fluctuations, the measured and modelled hourly VTEC for every day are added and averaged.

DTEC and percentage deviation $(p d)$ were also calculated to find mean difference and percentage. Following formulas are used to calculate DTEC and percentage deviation.

$$
\begin{aligned}
& \mathrm{d} \text { TEC }=\text { IRI TEC }- \text { GPS TEC } \\
& p d=\frac{(\text { IRITEC } i)-\left(G P S T E C_{i}\right)}{G P S T E C_{i}} \times 100
\end{aligned}
$$

\section{Results and Discussions}

The comparison was made between GPS and all IRI models over Sukkur region to identify the advantages of these models and for better use of these models in future over this region. Hour to hour variation in Figure 2 shows the relation between IRI models TEC and GPS TEC over Sukkur region in first three days of May 2019. Maximum TEC values were observed between the time of sunrise and sunset (about 08:00 hours to 07:00 hours) of local time in all three days.

Minimum values were observed (between 08:00 hours and 01:00 hours) of local time. It is affirmation that the solar radiation is a major cause of the ionization process in the ionosphere. The observations generally agree with well-known ionospheric theories on ionization. Correlation between GPS TEC and IRI models is shown in Table 1. GPS TEC is best correlated with IRI 2016 model (above 0.83) and least correlated with IRI 2007 (above 0.78).

Figure 3 is plot between different correlation values. Correlation of GPS with IRI 2012 and IRI 2016 are almost overlapping each other but correlation of GPS with IRI 2007 is little bit below in the graph showing that correlation of GPS with IRI 2012 and IRI 2016 are having good correlation coefficient. The Table 1 is showing only averaged value and Figure 3 is showing the plot of hourly data of first three days of May 2019.

In this paper, the data show very good correlation of GPS with IRI models specially IRI 2016 (above 0.83). Correlation of GPS with IRI 2012 is above 0.82 and correlation of GPS with IRI 2007 is above 0.78 . Figure 3.3 also showing the comparison of correlation values. Light discrepancies are due to upper integration limit, which was taken $1000 \mathrm{~km}$. As we know that upper integration limit is not constant, and the limits will not always correspond to $1000 \mathrm{~km}$ as we have used in this work. 
Deviation of IRI TEC and GPS TEC is shown in Figure 4. As per this graph IRI 2007 - GPS TEC is showing maximum values. It means that correlation of GPS TEC with IRI 2007 is less as compared to other models. We can also see that IRI 2016 is at lowest difference with GPS. Percentage deviation graph is an affirmation that IRI 2016 is at least percentage deviation (Figure 5). Maximum percentage deviation goes to IRI 2007 and IRI 2012 as compared to IRI 2016.

Comparison between Dst and $\mathrm{Kp}$ indices have been made in Figure 6. Dst and Kp index estimate the strength of the geomagnetic storm. Both values in above mentioned graph are showing the greater strength of storm from 7 pm May 1, 2019 to 5 am May 2, 2019. Moderate strength is shown at the daytime on May 2, 2019. An increase in strength of storm is showing in the closing hours of $3^{\text {rd }}$ May 2019.

Due to geomagnetic storms, oxygen atoms present in ionosphere are largely ionized and results in increase in $\mathrm{O} / \mathrm{N}_{2}$ ratio. Intensity of the storm was low on May 1, 2019 over Sukkur region and suddenly increases on May 2, 2019 as Figure 7 shows $\mathrm{O} / \mathrm{N}_{2}$ data of three consecutive days MAY 1, 2019 to May 3, 2019. Sukkur region is highlighted in red color. We can see that on May 1, 2019 there are no abnormal clouds. A clear disturbance is shown on May 2, 2019 over Sukkur region indicating the maximum strength of storm. However, normal values were retrieved on May 3, 2019 by the atmosphere

\section{Conclusion}

In this paper, we compared GPS TEC with IRI 2007, IRI 2012 and IRI 2016 models over Sukkur region to check the correctness and reliability of the data gained from GPS and IRI models. Data of first three consecutive days of May 2019 was examined through different parameters.

a) GPS TEC and IRI models are showing almost similar trend in all three days. A moderate storm is observed specially on May 2, 2019 as per different indices (Dst $=-30 \mathrm{n} \mathrm{T}$ and Kp*10 =40). Comparison shows maximum value at sunrise of each day and minimum values at night as an affirmation that sun has its greater impact on atmospheric parameters.

b) The results show that the IRI TEC values generally compared well with the GPS TEC values, with correlation coefficients as good as about 0.83 with IRI 2016, 0.82 with IRI 2012 and 0.78 with IRI 2007. Diurnal and hourly comparisons were generally better than the day-to-day comparisons since the IRI model is known to compare well with mean or median values.

c) It was observed that IRI 2016 is a good model that can provide almost accurate estimates of ionospheric delays of signals propagating over Pakistan. Light discrepancies are due to upper integration limit, which was taken $1000 \mathrm{~km}$. As we know that upper integration limit is not constant, and the limits will not always correspond to $1000 \mathrm{~km}$ as we have used in this work. Here is another possible reason for the discrepancies are in the relatively small data from the region considered in developing the model. 


\section{References}

Bilitza, D. (2001). International Reference Ionosphere 2000. 36(2), 261-275. https://doi.org/10.1029/2000RS002432

Bilitza, D., Hernández-Pajares, M., Juan, J. M., and Sanz, J. (1999). Comparison between IRI and GPS-IGS derived electron content during 1991-1997. Physics and Chemistry of the Earth, Part C: Solar, Terrestrial \& Planetary Science, 24(4), 311-319. https://doi.org/10.1016/S1464-1917(99)00004-5

Jin, S., Jin, R., and Li, D. (2017). GPS detection of ionospheric Rayleigh wave and its source following the 2012 Haida Gwaii earthquake. Journal of Geophysical Research: Space Physics, 122(1), 1360-1372. https://doi.org/10.1002/2016JA023727

Okoh, D., Eze, A., Adedoja, O., Okere, B., and Okeke, P. N. (2012). A comparison of IRI-TEC predictions with GPS-TEC measurements over Nsukka, Nigeria. 10(10). https://doi.org/10.1029/2012SW000830

Shah, M., Ahmed, A., Ehsan, M., Khan, M., Tariq, M. A., Calabia, A., and Rahman, Z. u. (2020a). Total electron content anomalies associated with earthquakes occurred during 1998-2019. Acta Astronautica, 175, 268-276. https://doi.org/10.1016/j.actaastro.2020.06.005

Shah, M., Aibar, A. C., Tariq, M. A., Ahmed, J., and Ahmed, A. (2020b). Possible ionosphere and atmosphere precursory analysis related to $\mathrm{Mw}>6.0$ earthquakes in Japan. Remote Sensing of Environment, 239, 111620. https://doi.org/10.1016/j.rse.2019.111620

Shah, M., Inyurt, S., Ehsan, M., Ahmed, A., Shakir, M., Ullah, S., and Iqbal, M. S. (2020c). Seismo ionospheric anomalies in Turkey associated with $\mathrm{Mw} \geq 6.0$ earthquakes detected by GPS stations and GIM TEC. Advances in Space Research, 65(11), 25402550. https://doi.org/10.1016/j.asr.2020.03.005

Tariq, M. A., Shah, M., Hernández-Pajares, M., and Iqbal, T. (2019). Ionospheric VTEC variations over Pakistan in the descending phase of solar activity during 2016-17. Astrophysics and Space Science, 364(6), 1-12. https://doi.org/10.1007/s10509-019$\underline{3591-3}$

Tariq, M. A., Shah, M., Inyurt, S., Shah, M. A., and Liu, L. (2020). Comparison of TEC from IRI-2016 and GPS during the low solar activity over Turkey. Astrophysics and Space Science, 365(11), 1-13. https://doi.org/10.1007/s10509-020-03894-3 


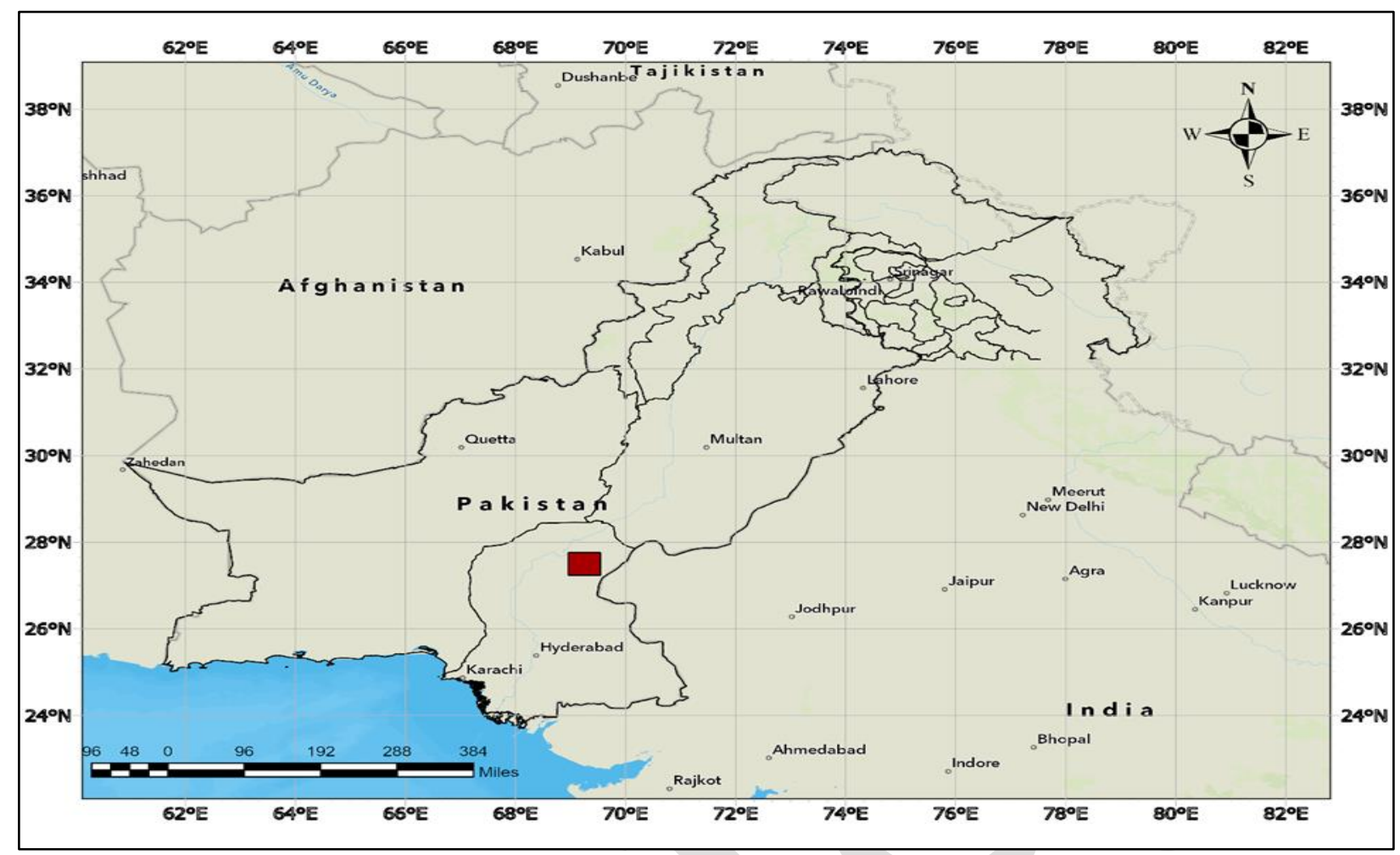

Figure 1: Geographical location of Sukkur, Pakistan

\section{COMPARISON OF GPS TEC WITH IRI MODELS OVER SUKKER REGION}

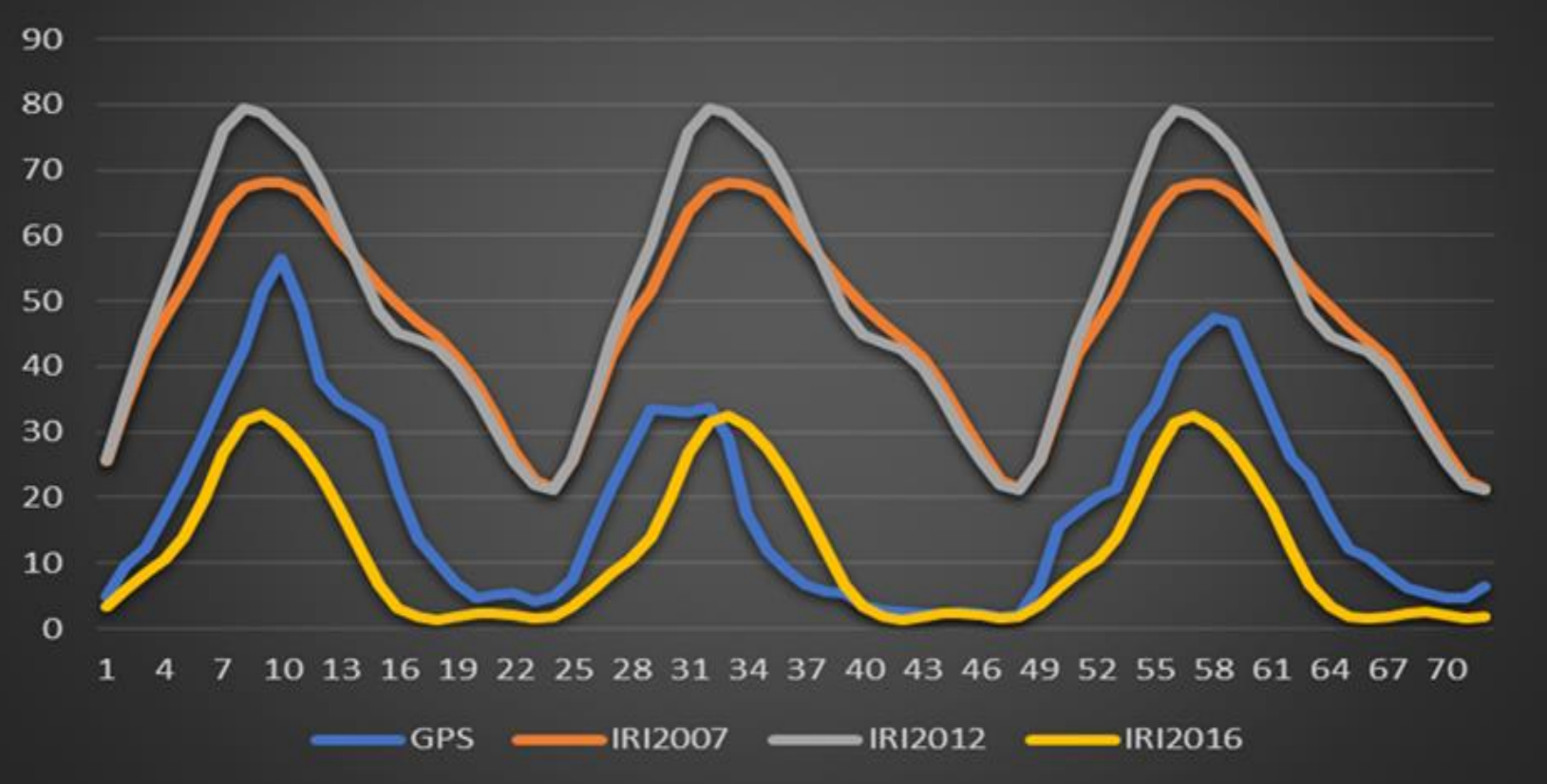

Figure 2: Comparison of GPS TEC with IRI models on MAY 1, 2 and 3, 2019 over Sukkur region 
Table-1: Correlation values of GPS TEC with IRI models

\begin{tabular}{|c|c|c|}
\hline $\begin{array}{c}\text { Sr. } \\
\text { No. }\end{array}$ & Correlation of GPS & Correlation value (AVERAGED) \\
\hline A & WITH IRI 2007 & 0.787797 \\
\hline B & WITH IRI 2012 & 0.827017 \\
\hline C & WITH IRI 2016 & 0.830174 \\
\hline
\end{tabular}

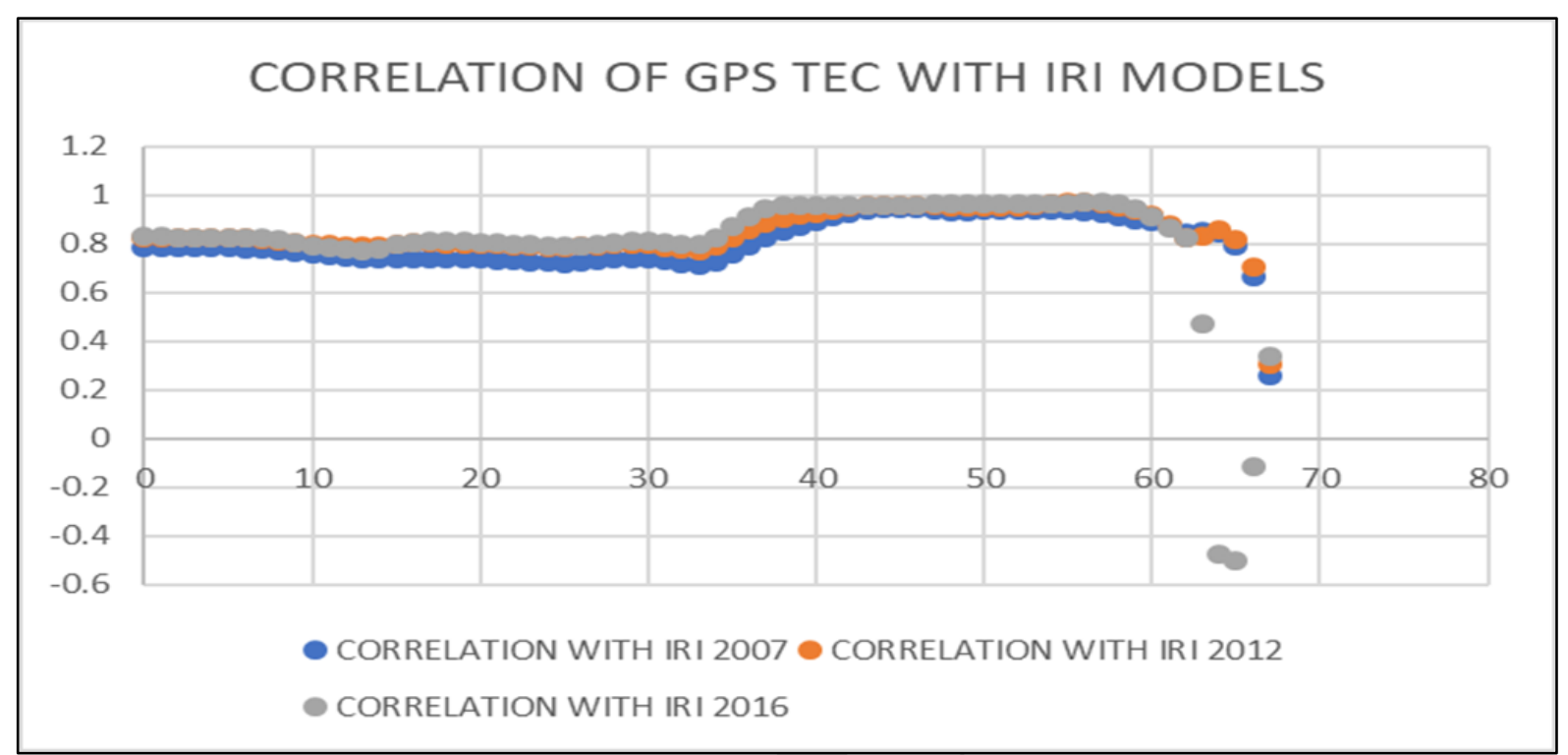

Figure 3: Hourly correlation of GPS TEC with IRI model. Horizontal axis is showing hours of days and vertical axis is showing correlation values

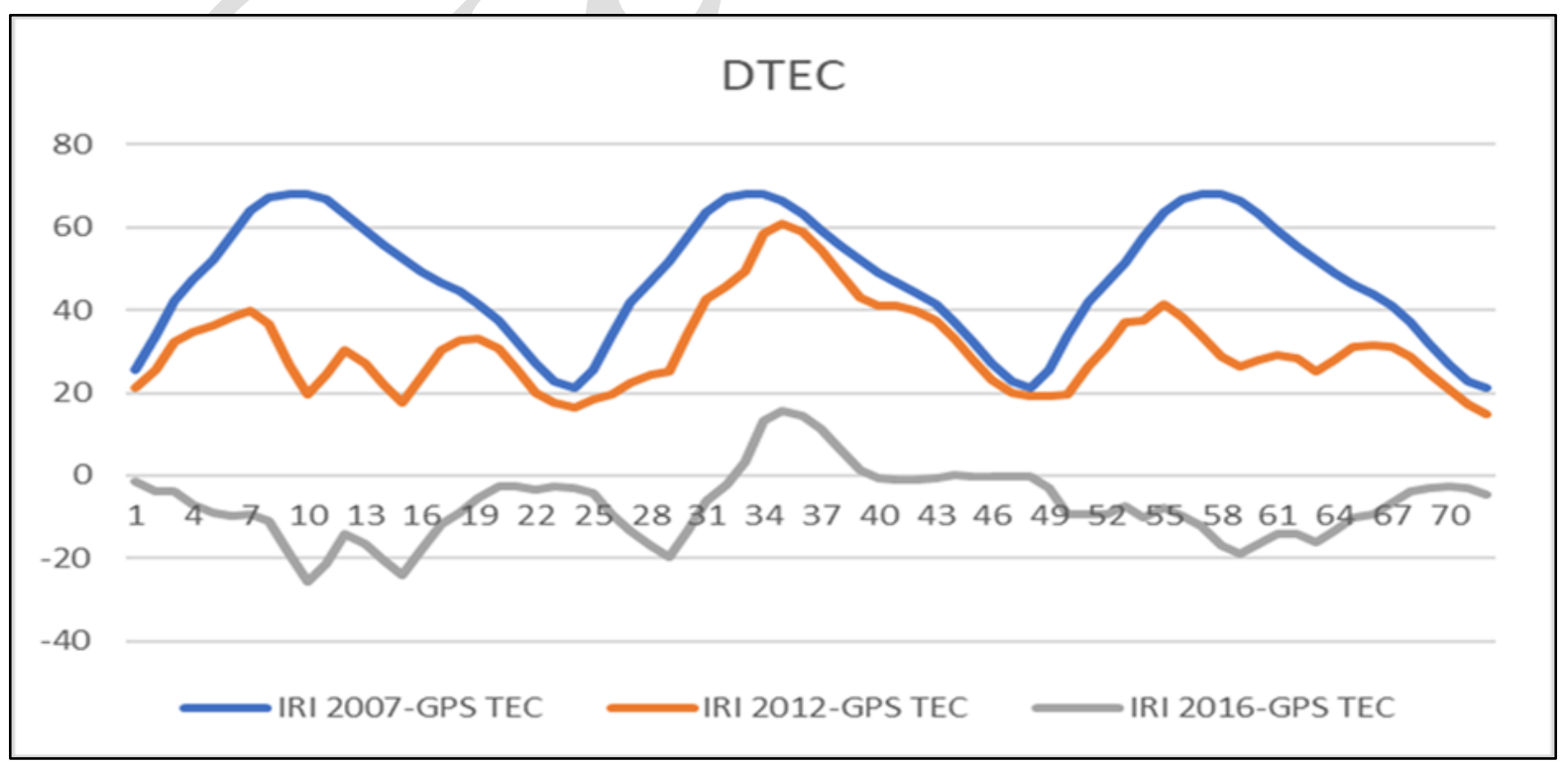

Figure 4: DTEC (IRI - GPS). Horizontal axis is showing hours of days and vertical axis is showing DTEC 


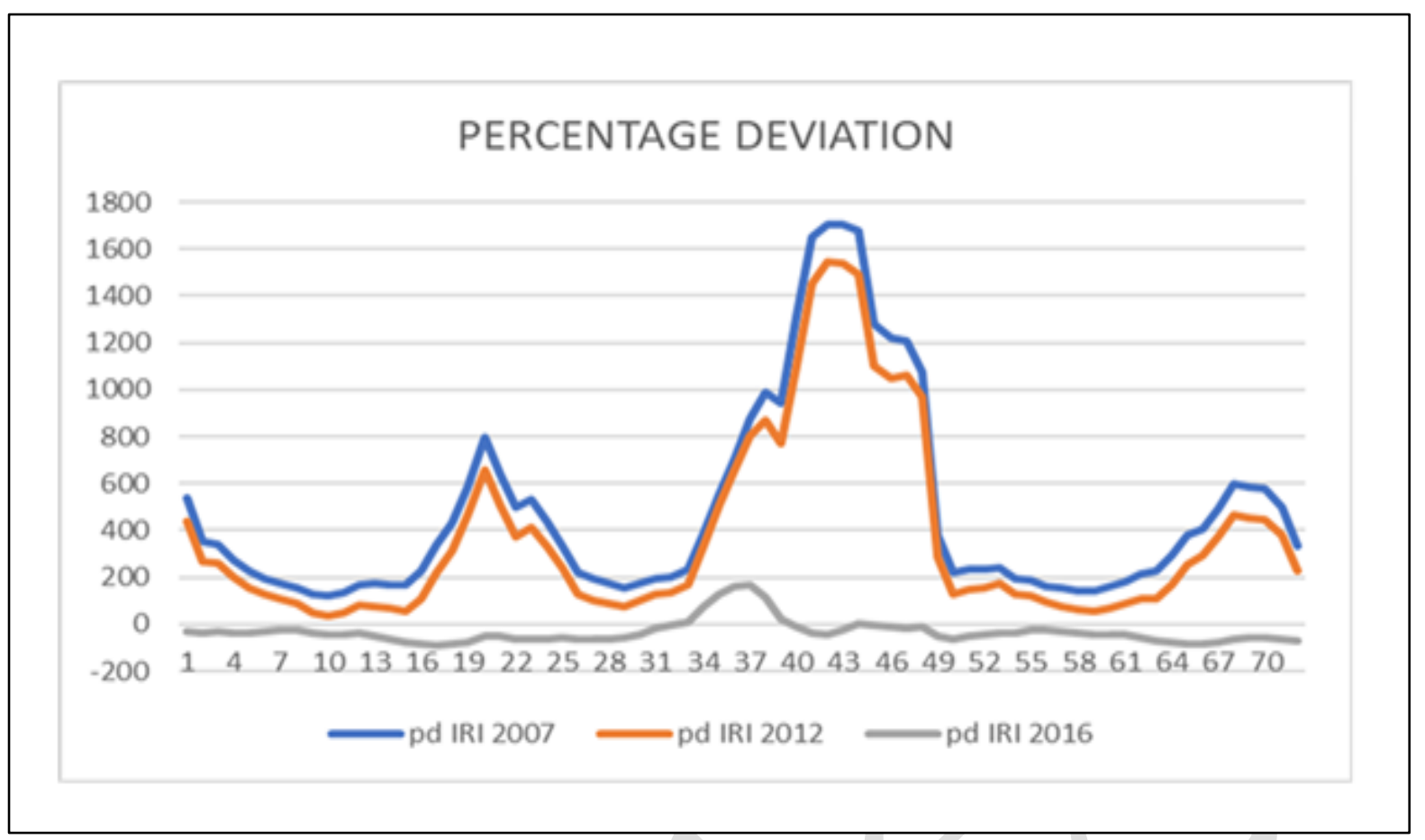

Figure 5: Percentage Deviation

Horizontal axis is showing hours of days and vertical axis is showing percentage deviation.

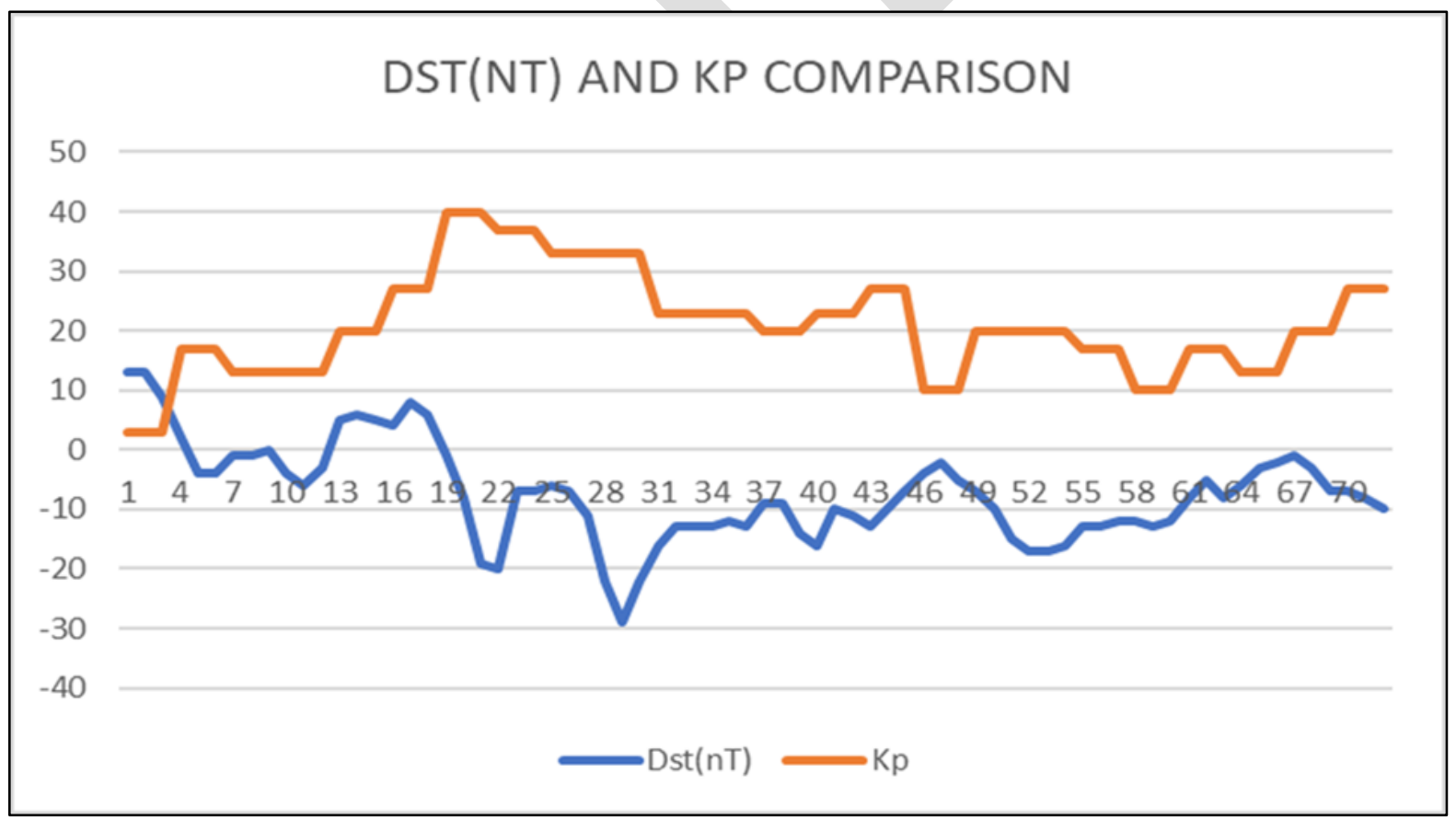

Figure 6: Dst and Kp indices comparison. Horizontal axis is showing hours of days and vertical axis is showing Dst and $\mathrm{Kp}$ indices values 


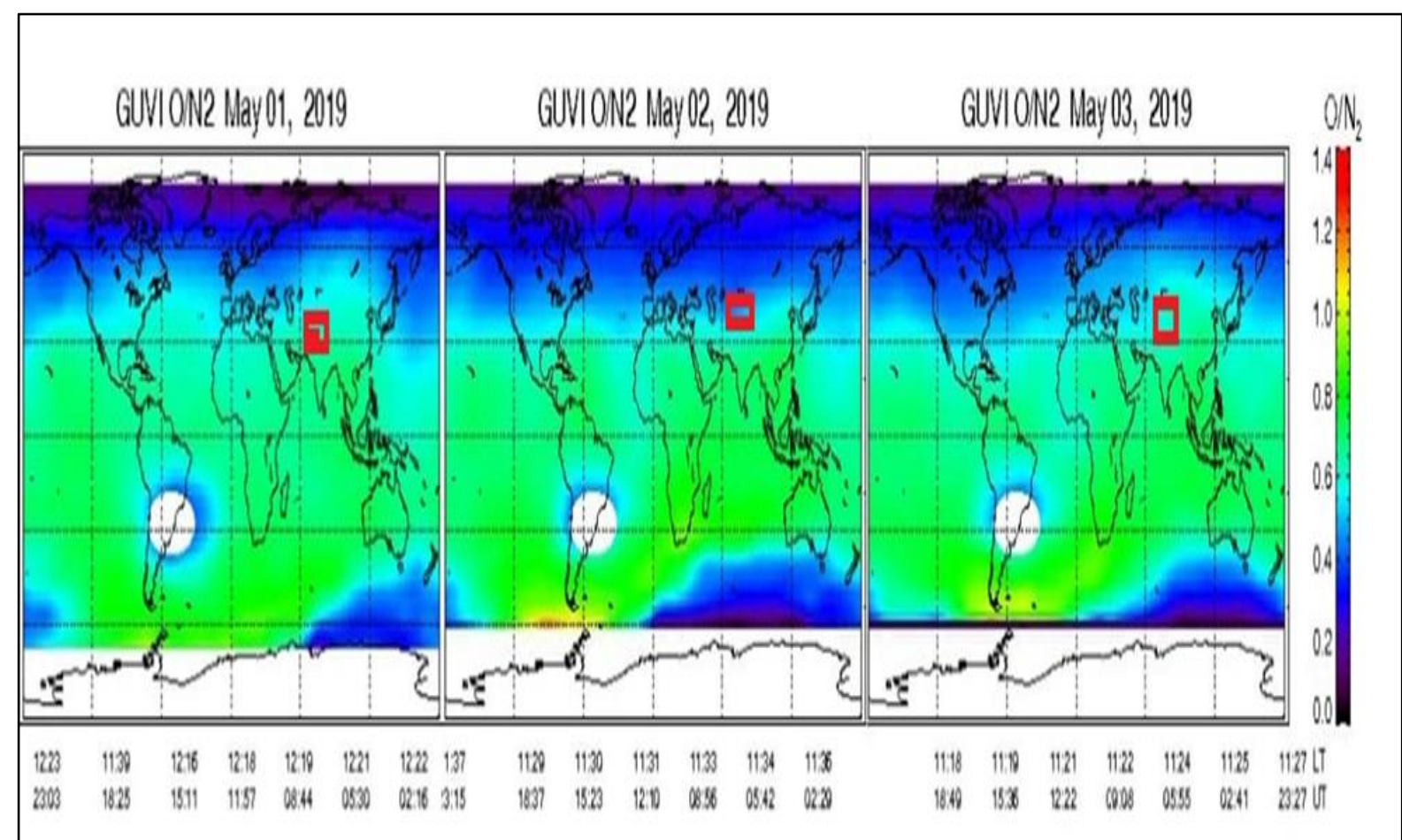

Figure 7: O/N_2 (column density ratio). Figure showing abnormal clouds over Sukkur on May 2, 2019 